\title{
The Processes Driving an Ember Storm
}

\author{
John Dold, ${ }^{a}$ Kathleen Scott ${ }^{\mathrm{a}}$ and James Sanders ${ }^{\mathrm{a}}$

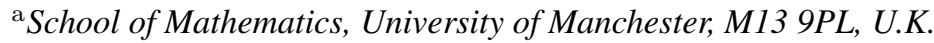 \\ Email: John.Dold@manchester.ac.uk
}

\begin{abstract}
A form of fire spread that has sometimes been called an 'ember storm' is examined, in which the main vector of fire spread is the ignition of numerous spotfires by embers or firebrands. By taking spotfires to grow as elliptical shapes under the influence of wind, the processes from ember production to spotting can be assembled into an averaged continuum model. The model is not valid in the limit of relatively rare spotting but for fires in which spreading involves frequent ignition of new spotfires it amounts to relatively simple bookkeeping of the mean number of spotfires, their perimeter, the area burnt and the transport and deposition of lofted embers. The basic ingredients in the model are outlined and we examine the way in which the average spread rate of an ember storm depends on such things as the rate of ember production within the flaming perimeter, the transport of embers and their propensity for igniting fresh vegetation when they land. Key factors are the wind-speed and what may be called the mean spotting time, or the time it takes for any one spotfire to lead to the ignition of another. A single similarity solution describes steady spread over all parameter ranges, requiring one numerical calculation to predict all steady ember storm spread rates, when dominated by frequent spotting.
\end{abstract}

In more detail, two dimensionless quantities are revealed in the examination of the model. These are

$$
\tau=\frac{t_{\mathrm{e}}}{t_{\mathrm{s}}}=\frac{\text { mean ember lifetime }}{\text { mean spotting time }} \quad \text { and } \quad \lambda=\frac{\ell_{\mathrm{e}}}{\ell_{\mathrm{s}}}=\frac{\text { mean ember transport distance }}{\text { mean ember storm thickness }}
$$

in which the mean spotting time $t_{\mathrm{s}}$ is the average time it takes for one spotfire to grow and produce enough embers to generate a new spotfire; the mean ember storm thickness $\ell_{\mathrm{s}}$ is the characteristic distance that the overall ember storm moves, ahead of the centroid movement of individual spotfires, during the spotting time. The ember lifetime $t_{\mathrm{e}}$ is the characteristic survival time of lofted embers (lost through extinction and landing) and the mean ember transport distance $\ell_{\mathrm{e}}$ is the distance that they are transported by the wind, during this time, ahead of the mean movement of the ember storm.

For a steadily spreading ember storm the dimensionless ember transport distance $\lambda$ takes a characteristic value of $\lambda=\bar{\lambda} \approx 0.305 \cdots$. The mean spread rate of the ember storm is then given by

$$
R_{\mathrm{e}}=\frac{\tau w+\bar{\lambda} c}{\tau+\bar{\lambda}} \approx \frac{w t_{\mathrm{e}}}{t_{\mathrm{e}}+0.3 t_{\mathrm{s}}}
$$

where $w$ is the effective mean wind speed and $c$ is the mean speed of the centroid of a spotfire. This result should hold well when $\tau w$ is large compared with the centroid speed $c$, this being a condition that ensures that numerous spotfires are formed and that these dominate the spread of the fire- this is required for an ember storm to be present. Otherwise, spotting is more rare and it generates more occasional additions to the forward motion of the fire; results for mean fire spread with occasional spotting will be addressed in a separate publication.

Keywords: Ember Storm, Firebrand, Spotfire, Fire Spread, Wind driven fire. 


\section{INTRODUCTION}

In addition to the continuous, often elliptical spread of a fireline or fire perimeter (Anderson et al., 1982; Richards, 1990) bushfires that are intense enough to lift burning embers or firebrands in their rising buoyant flow of hot combustion products (Tarifa et al., 1965; Woycheese et al., 1999; Ellis, 2000; Anthenian et al., 2006) are potentially able to leap forwards through the generation of new fires or spotfires. If an ember is still burning when it lands in sufficiently light and dry vegetation, it is capable of generating a new fire (Manzello et al., 2008) which, in turn, can go on to generate more burning embers and new spotfires. Such fires can be a cause of great difficulty in fire suppression (McRae, 2010) requiring rapid response to avoid an accumulation in the number of fires having to be dealt with. Further references on aspects of this process can be found, for example, in Consalvi et al. (2011).

In some cases spotfire generation can be the primary mechanism by which a fire spreads. The term ember storm has been used in this context (McRae, 2010) and will be adopted here to describe such a fire. Ember storms are known to offer the most dangerous conditions for the transmission of burning from bushfires or wildfires into structural or building fires (Chen and McAneney, 2004; Gill, 2005).

In an ember storm, a fire generates so many embers and spotfires that the overall fire spread is no longer a small deviation from the usual spread of a head fire. Its effective or average rate of spread must be determined by a range of related factors such as the growth of individual spotfires, the rate of ember production by each of these fires, the transport, extinction and landing rates of individual embers and the propensity for any ember to ignite a new spotfire.

Little work has been done in examining the combined effect of these processes in determining the overall fire spread; an article by Porterie et al. (2005) takes a step in this direction by using a 'small-world' numerical simulation approach. As this article shows, a remarkable degree of simplicity is revealed through a continuum model for the processes driving an ember storm. Straightforward conservation equations for four quantities, the number of lofted embers, the number of individual spotfires, the fire perimeter and the burnt area (all measured as averages per unit area of ground) are found to reveal how the ember storm and its mean spread rate are related to each of the underlying contributing factors.

\section{CONTINUUM MODEL FOR AN EMBER STORM}

Some of the core concepts that need to be included in a generic description for an ember storm are illustrated in Figure 1. All terms used in the modelling are listed in Table 1.

\subsection{Model equations}

Embers land and ignite new spotfires that have a preferential direction of spread (that of the mean wind). At any time, a unit area can contain a mean number of $n$ such fires, in which for convenience we also

Table 1: Nomenclature of terms used in this article with a brief description of their physical significance.

\begin{tabular}{|cll|cl|}
\hline$E$ & lofted burning embers per unit area & $n$ & potential ignitions per unit area \\
$\epsilon$ & embers produced per area burnt & $b$ & sum of all $n$ fire areas per unit area \\
$\zeta$ & landing rate of lofted embers & & (without deducting overlaps) \\
$\eta$ & extinction rate of lofted embers & $f$ & sum of all $n$ fire perimeters per unit area \\
$\kappa$ & ignition probability per burning ember & & (without deducting overlaps) \\
$w$ & mean wind speed (in the $x$-direction) & $B$ & proportion of area actually burnt \\
$R_{\mathrm{H}}$ & head fire spread rate & $F$ & actual fire perimeter per unit area \\
$R_{\mathrm{F}}$ & flank fire spread rate & $t$ & time, $\quad x \quad$ spatial distance \\
$R_{\mathrm{B}}$ & backing fire spread rate & $t_{\mathrm{S}}$ & mean spotting time per spotfire \\
$R_{\mathrm{e}}$ & overall spread rate of the ember storm & $\ell_{\mathrm{S}}$ & mean ember storm thickness \\
$S$ & mean spread rate for fire perimeter & $t_{\mathrm{e}}$ & mean lifetime of lofted burning embers \\
$s$ & mean spread rate for fire area & $\ell_{\mathrm{e}}$ & mean ember transport distance \\
$c$ & centroid speed of elliptical spotfires & $t_{\mathrm{i}}$ & spotfire ignition time per lofted ember \\
\hline
\end{tabular}




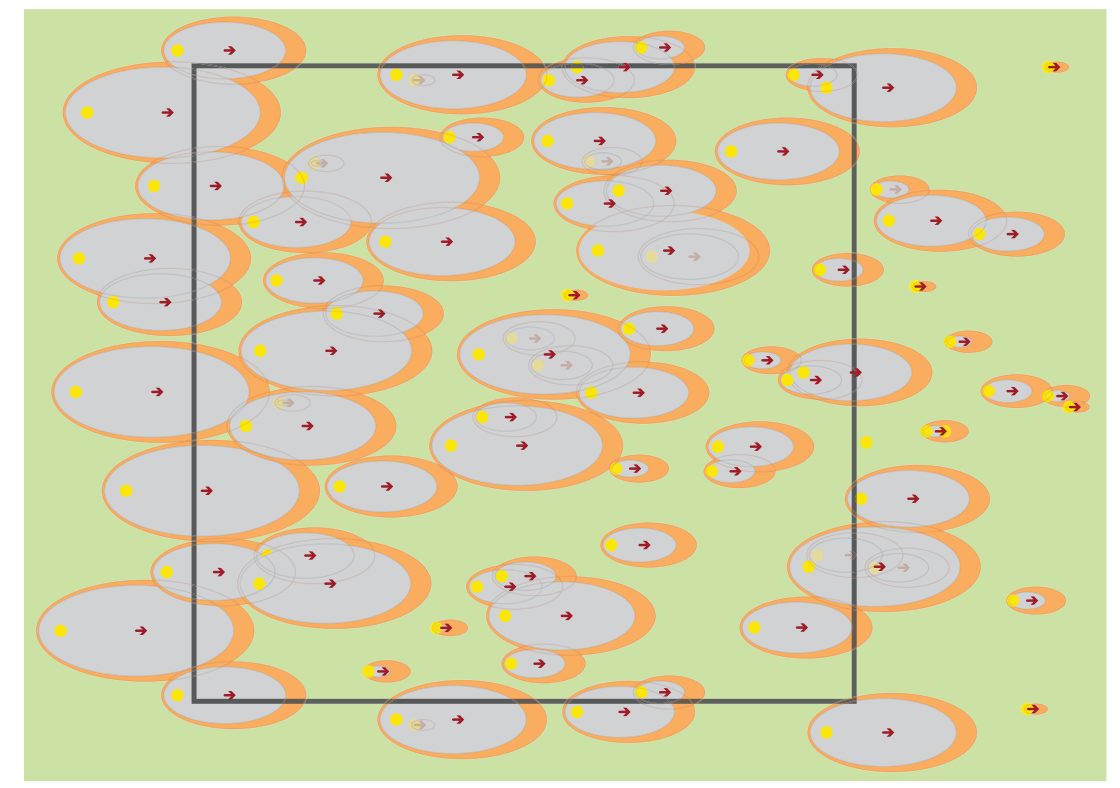

Figure 1: Elliptical fires of equal aspect ratio and orientation, distributed randomly in size and position; yellow dots represent $n$ ignitions per unit area (the rectangle) including would-be or virtual ignitions if the ember had not landed in a region already burnt; the orange areas mark out the vegetation that is engaged in the fire over a short time interval during which the arrows mark the movement of each centroid. Statistics for covering areas with randomly placed and sized objects can be applied, taking into account the geometrical properties of elliptical fire growth.

count virtual spotfires that would have ignited had they not landed in an area that is already burnt; it is straightforward to account for such exceptions, as will be seen later. The value of $n$ therefore grows with time as more embers land, taking each landing burning ember to have a positive probability $\kappa$ of causing an ignition. The statistics for virtual ignitions is taken to be the same as for real ignitions even though embers approaching burnt areas might well be deflected differently by flames and plumes. However, this makes no difference to the end result in which virtual fires do not contribute.

Those fires that were ignited earlier have a larger area and perimeter, in which it is again convenient to ignore overlaps in burnt areas. These contribute to a burnt area $b$ and a fire perimeter $f$, averaged per unit area and including all fire spread as if it continued uninterupted through already burnt regions. Because overlaps are not deducted from such a representation, the growth rates of $f$ and of $b$ are given simply by

$$
f_{t}+c f_{x}=2 \pi S n \quad \text { and } \quad b_{t}+c b_{x}=s f
$$

where $S$ and $s$ are equivalent spread rates for nominally circular fires, having a perimeter that grows in sole proportion to the number of fires $n$ and an area that grows in sole proportion to the perimeter $f$, for constant values of $S$ and $s$. The presence of the 'convective' term $c \partial_{x}$ in these equations is needed because the centroid of each spotfire moves at a speed $c$ in the direction of the wind. Effectively, the left hand sides of these equations represent the time derivatives of $f$ and $b$ in a reference frame that moves to the right at the characteristic speed $c$. Along these characteristics, the growth rates are given by the right hand sides of the equations.

For elliptical fires with a wind-speed dependent head fire spread rate of $R_{\mathrm{H}}$, flank fire spread-rate $R_{\mathrm{F}}$ and backing fire spread rate $R_{\mathrm{B}}$, each individual spotfire can be taken to grow as an ellipse of aspect ratio $r=2 R_{\mathrm{F}} /\left(R_{\mathrm{H}}+R_{\mathrm{B}}\right)$ having the perimeter and area at a time $t$ after ignition

$$
\begin{aligned}
& f: \quad 2 \pi t S=2 t\left(R_{\mathrm{H}}+R_{\mathrm{B}}\right) \int_{0}^{\pi / 2} \sqrt{1-\left(1-r^{2}\right) \cos \theta} \mathrm{d} \theta \\
& b: \quad \pi t^{2} S s=\pi t^{2} \frac{1}{2}\left(R_{\mathrm{H}}+R_{\mathrm{B}}\right) R_{\mathrm{F}}
\end{aligned}
$$



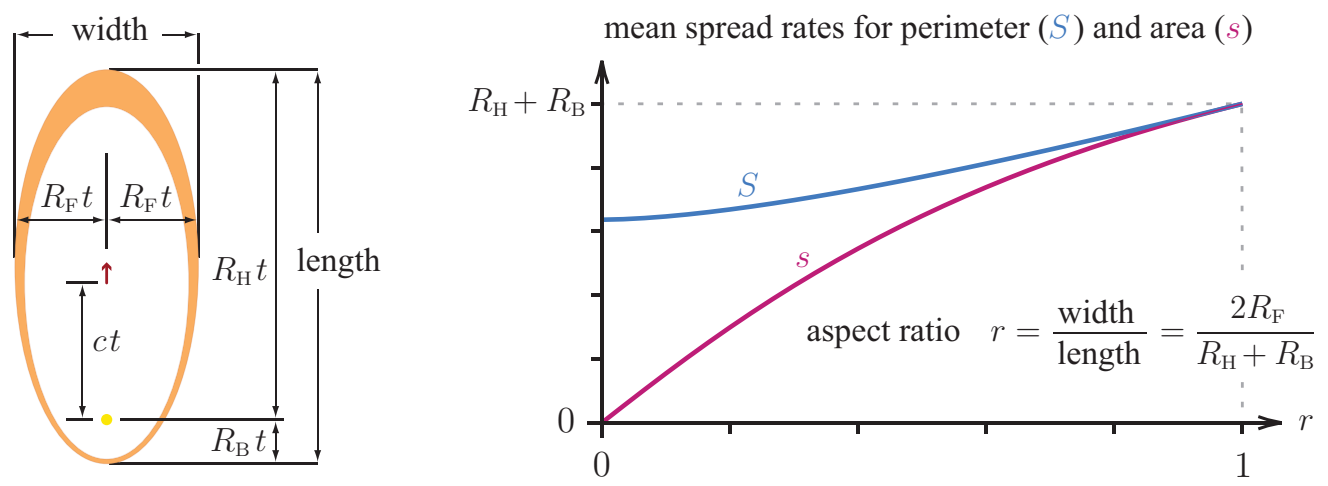

Figure 2: The geometric shape of an elliptical fire (left) with head fire spread rate $R_{\mathrm{H}}$, backing fire spread rate $R_{\mathrm{B}}$ and flank fire spread rate $R_{\mathrm{F}}$. The fire is ignited at the yellow dot and the red arrow marks the movement of the centroid (speed $c$ ) during the time that the front of the fire sweeps through the orange area. The graph on the right gives the values for two different definitions of mean spread-rate, averaged over an elliptical fire of aspect ratio $r$, that describe either the rate of increase in burnt area per unit length of fire perimeter $(s)$ or the rate of increase in fire perimeter with time $(S)$. For unit aspect ratio $r$ the fires grow as pure circles with $S=s=R_{\mathrm{F}}=R_{\mathrm{B}}=R_{\mathrm{H}}$ and $c=0$; more generally $s<S$.

from geometrical considerations applied to ellipses. Time can be divided from the equalities in (4) to reveal formulae for $S$ and $s$ that depend only on the head, flank and backing spread rates of the elliptical fires. Numerical curve-fitting to the integral in (4) leads to the very good approximations

$$
\begin{aligned}
S & =\frac{1}{4}\left(R_{\mathrm{H}}+R_{\mathrm{B}}\right)(3(1+r)-\sqrt{(3+r)(1+3 r)})\left(1+\frac{1}{246} e^{-16 r}\right) \pm \frac{1}{100} \% \\
s & =\frac{1}{2}\left(R_{\mathrm{H}}+R_{\mathrm{B}}\right) R_{\mathrm{F}} / S \\
c & =\frac{1}{2}\left(R_{\mathrm{H}}-R_{\mathrm{B}}\right)
\end{aligned}
$$

in which we also identify the mean centroid speed $c$ of each elliptical fire. Values of $S$ and $s$ are compared with the sum of head and backing fire spread-rates $R_{\mathrm{H}}+R_{\mathrm{B}}$ in Figure 2, for all possible aspect ratios $r$.

Spotfires that do not grow as ellipses, but as some other self-similar shape, could be treated in a similar way, with analogous geometrical considerations. Qualitatively similar formulae would arise with appropriate values arising for $S, s$ and $c$. As in the case of elliptical fire spread, these would ultimately depend on the wind speed $w$ for any given vegetation conditions.

The rate at which new potential ignitions occur depends on the rate at which burning embers land per unit area. Taking the lofted burning ember density to be $E$, above unit ground area, and the landing rate to be $\zeta$, the rate at which new spotfires form is simply

$$
n_{t}+c n_{x}=\kappa \zeta E=E / t_{\mathrm{i}}
$$

with $\kappa$ being the probability that a burning ember ignites a spotfire on landing in unburnt vegetation, having $\kappa \zeta=1 / t_{\mathrm{i}}$ for a characteristic spotfire ignition time per lofted ember of $t_{\mathrm{i}}$.

To close the problem it is necessary to model the production, transport, extinction and landing of embers. The production rate of embers needs consideration not of $f$, the fire perimeter that does not exclude overlaps, but of the actual burning fire perimeter $F$. Given this quantity, the rate at which vegetation is actually burnt per unit area is $s F$. Thus if $\epsilon$ embers are produced per unit area of vegetation burnt then the rate of production of embers per unit area becomes simply $\epsilon s F$. If embers are transported at a mean or effective wind speed of $w$, the conservation law for lofted burning embers becomes

$$
E_{t}+w E_{x}=\epsilon s F-(\eta+\zeta) E=\epsilon s F-E / t_{\mathrm{e}}
$$

where $\eta$ represents the rate at which embers are extinguished while still lofted, having $\eta+\zeta=1 / t_{\mathrm{e}}$ for a characteristic ember lifetime, or equivalently an ember loss-time, of $t_{\mathrm{e}}$. 
At this point a core assumption is needed to link $f$ and $F$. We shall also link $B$, the actual area burnt per unit area with overlaps only counted once, and $b$ the area burnt where overlaps count more than once. In this article we assume that the burnt proportion $B$ changes slowly with distance $x$, on a length scale that is large compared with the average size of each spotfire, as illustrated in Figure 1. This assumption is consistent with the idea of an ember storm, in which many spotfires form and embers are transported over at least several times the characteristic size of spotfires before they land and ignite new spotfires. In situations where this assumption is not valid, a modified approach is needed that is not described here.

In this context, we can consider increasing the potential burnt area $b$ by a small amount $\mathrm{d} b$. If the proportion actually burnt is $B$ then the probability of increasing $B$ is proportional to the area that remains unburnt, namely $1-B$. The increase in $B$ is therefore

$$
\mathrm{d} B=(1-B) \mathrm{d} b \quad \text { so that } \quad 1-B=e^{-b}
$$

in which the latter relation between $B$ and $b$ follows from a simple integration. Likewise, the probability that any part of the perimeter $f$ is not in an already burnt area is also $1-B$; it follows that

$$
F=(1-B) f=e^{-b} f
$$

Eliminating the unphysical quantities $b$ and $f$ and writing the conservation equations in a reference frame that moves to the right (the direction of ember-storm propagation) at a speed $R_{\mathrm{e}}$, that is intended to represent the mean spread rate of the ember storm, reveals the model system

$$
\begin{aligned}
n_{t}-\left(R_{\mathrm{e}}-c\right) n_{x} & =\kappa \zeta E & F_{t}-\left(R_{\mathrm{e}}-c\right) F_{x} & =2 \pi S n(1-B)-s F^{2} /(1-B) \\
B_{t}-\left(R_{\mathrm{e}}-c\right) B_{x} & =s F & E_{t}+\left(w-R_{\mathrm{e}}\right) E_{x} & =\epsilon s F-(\eta+\zeta) E .
\end{aligned}
$$

An ember storm travelling to the right would also require the far-field boundary conditions

$$
\lim _{x \rightarrow \infty}(E, F, n, B)=(0,0,0,0) \quad \text { and } \quad \lim _{x \rightarrow-\infty}\left(E, F, n_{x}, B\right)=(0,0,0,1)
$$

with the proportion of area burnt $B$ increasing from zero to one through the ember storm.

\subsection{Characteristic quantities and dimensionless form of the model}

There is a natural characteristic time inherent in this set of equations, that can be written as

$$
t_{\mathrm{S}}=\left(\frac{\eta+\zeta}{\pi s S \varepsilon \kappa \zeta}\right)^{1 / 2}=\left(\frac{2 t_{\mathrm{i}}}{\pi\left(R_{\mathrm{H}}+R_{\mathrm{B}}\right) R_{\mathrm{F}} \varepsilon t_{\mathrm{e}}}\right)^{1 / 2}
$$

the final version of which does not require knowledge of $S$ and $s$. To better understand this formula for $t_{\mathrm{s}}$, it can be seen that $\pi s S t_{\mathrm{s}}^{2}$ is the area of an elliptical spotfire at a time $t_{\mathrm{s}}$ after its ignition, when it has lofted a total of $\pi s S t_{\mathrm{s}}^{2} \varepsilon$ embers. A proportion $\zeta /(\eta+\zeta)$ of these embers falls back to ground still burning and a further proportion $\kappa$ of these is able to start new fires. Since $\pi s S t_{\mathrm{s}}^{2} \varepsilon \kappa \zeta /(\eta+\zeta)=1$, this means that the fire that has grown in the time $t_{\mathrm{s}}$ ignites exactly one new spotfire, on average. Thus $t_{\mathrm{s}}$ is the average spotting time for any one elliptical fire, being the time in which a new fire is formed from any one elliptically growing mini-fire. This is a key time-scale associated with the evolution of an ember storm. During the spotting time $t_{\mathrm{s}}$ the ember storm moves a distance of

$$
\ell_{\mathrm{s}}=\left(R_{\mathrm{e}}-c\right) t_{\mathrm{s}}
$$

beyond the characteristic centroid movement of individual fires, which can thus be thought of as measuring an average ember storm thickness, based on the spotting time $t_{\mathrm{s}}$.

Also, since $\eta+\zeta$ is the rate at which lofted burning embers are lost, its reciprocal $t_{\mathrm{e}}=1 /(\eta+\zeta)$ represents the mean lifetime of lofted burning embers. Relative to the reference frame moving at the ember-storm spread rate $R_{\mathrm{e}}$ these embers are transported a mean distance of $\ell_{\mathrm{e}}=\left(w-R_{\mathrm{e}}\right) t_{\mathrm{e}}$ as they are carried by the wind. The pairs of characteristic time and length scales lead to two dimensionless ratios

$$
\tau=\frac{t_{\mathrm{e}}}{t_{\mathrm{s}}}=\frac{1}{(\eta+\zeta) t_{\mathrm{s}}} \quad \text { and } \quad \lambda=\frac{\ell_{\mathrm{e}}}{\ell_{\mathrm{s}}}=\frac{w-R_{\mathrm{e}}}{R_{\mathrm{e}}-c} \tau
$$



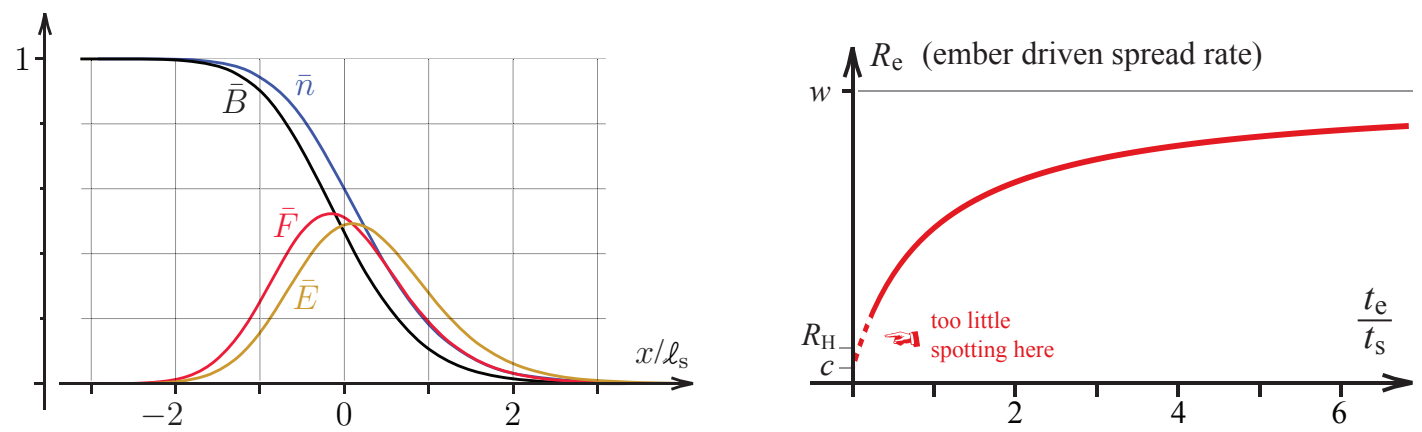

Figure 3: Left: steady dimensionless profiles for ember density $\bar{E}$, number of ignitions $\bar{n}$, fire perimeter $\bar{F}$ and area burnt $\bar{B}$, all per unit area, plotted as functions of distance measured in multiples of the spotting distance $\ell_{\mathrm{S}}$; the origin for $x / \ell_{\mathrm{S}}$ in this graph is chosen arbitrarily. Right: illustration of the dependence of ember storm spread rate on the ratio $\tau=t_{\mathrm{e}} / t_{\mathrm{s}}$.

with $\tau$ representing the ember lifetime $t_{\mathrm{e}}$ measured in units of the spotting time $t_{\mathrm{s}}$ and $\lambda$, the ember transport distance $\ell_{\mathrm{e}}$ in units of the ember storm thickness $\ell_{\mathrm{s}}$. If the variables of the problem are all now rescaled, or measured in units of characteristic dimensional values of $x, t, E, F$ and $n$, in the manner

$$
x=\ell_{\mathrm{s}} x^{\prime} \quad t=t_{\mathrm{s}} t^{\prime} \quad E=\frac{\epsilon}{(\eta+\zeta) t_{\mathrm{s}}} E^{\prime} \quad F=\frac{1}{s t_{\mathrm{s}}} F^{\prime} \quad n=\frac{\epsilon \kappa \zeta}{\eta+\zeta} n^{\prime}
$$

apart from the burnt proportion $B$ which is already dimensionless, the equation system takes the form

$$
\begin{aligned}
n_{t^{\prime}}^{\prime}-n_{x^{\prime}}^{\prime} & =E^{\prime} & F_{t^{\prime}}^{\prime}-F_{x^{\prime}}^{\prime} & =2 n^{\prime}(1-B)-F^{2} /(1-B) \\
B_{t^{\prime}}-B_{x^{\prime}} & =F^{\prime} & \tau E_{t^{\prime}}^{\prime}+\lambda E_{x^{\prime}}^{\prime} & =F^{\prime}-E^{\prime}
\end{aligned}
$$

featuring both of the dimensionless parameters $\tau$ and $\lambda$.

\subsection{Steady spread of an ember storm}

If an ember storm is spreading at a steady speed $R_{\mathrm{e}}$ then the formulation of the problem requires that all time derivatives become zero. The dimensionless model equations are then

$$
n_{x^{\prime}}^{\prime}=-E^{\prime}, \quad B_{x^{\prime}}=-F^{\prime}, \quad F_{x^{\prime}}^{\prime}=F^{\prime 2} /(1-B)-2 n^{\prime}(1-B), \quad \lambda E_{x^{\prime}}^{\prime}=F^{\prime}-E^{\prime}
$$

in which only the parameter $\lambda$ survives. Moreover, these equations are subject to the boundary conditions

$$
\lim _{x^{\prime} \rightarrow \infty}\left(E^{\prime}, F^{\prime}, n^{\prime}, B\right)=(0,0,0,0) \quad \text { and } \quad \lim _{x^{\prime} \rightarrow-\infty}\left(E^{\prime}, F^{\prime}, n_{x^{\prime}}^{\prime}, B\right)=(0,0,0,1)
$$

so that the system has the form of a nonlinear eigenvalue problem, with eigenvalue $\lambda$. Numerical examination of the problem reveals that such a steady solution exists only for one value of $\lambda$, namely

$$
\lambda=\bar{\lambda}=0.3051 \cdots
$$

giving curves for the steady solutions $E^{\prime}=\bar{E}\left(x^{\prime}\right), F^{\prime}=\bar{F}\left(x^{\prime}\right), n^{\prime}=\bar{n}\left(x^{\prime}\right)$ and $B=\bar{B}\left(x^{\prime}\right)$ shown in Figure 3, where the origin for $x^{\prime}$ is arbitrary since (16)-(18) are unchanged by adding any constant to $x^{\prime}$.

That is, the spread-rate $R_{\mathrm{e}}$ adjusts itself so that the ember storm thickness $\ell_{\mathrm{s}}$ and ember transport distance $\ell_{\mathrm{e}}$ adopt the fixed ratio $\ell_{\mathrm{e}}: \ell_{\mathrm{S}}=\bar{\lambda}: 1$. Solving equations (13) and (14) then gives the formula

$$
R_{\mathrm{e}}=\frac{w \tau+c \bar{\lambda}}{\tau+\bar{\lambda}}=\frac{w t_{\mathrm{e}}+c \bar{\lambda} t_{\mathrm{s}}}{t_{\mathrm{e}}+\bar{\lambda} t_{\mathrm{s}}}
$$

for the ember storm spread rate, the variation of which is also sketched in Figure 3. In making use of this formula, it can be noted that the lifetime of lofted burning embers $t_{\mathrm{e}}$ should be insensitive to wind-speed, depending only on the rates of extinction and of landing of embers. The spotfire self-replication time $t_{\mathrm{s}}$ seen in equation (12) does depend on the wind speed $w$ through the head, backing and flank fire spread rates of each spotfire. Overall $t_{\mathrm{s}}$ should decrease as $w$ increases, causing the ember storm spread rate $R_{\mathrm{e}}$ to approach the wind-speed $w$. 


\section{Conclusions}

An ember storm is driven by a number of interacting processes. As adopted in the modelling here, the core processes are: self-similar elliptical growth of spotfires, involving $R_{\mathrm{H}}, R_{\mathrm{F}}$ and $R_{\mathrm{B}}$; a mean ember production rate $\epsilon$ per unit burnt area; ember transport at a mean effective wind speed $w$; mean rates of landing $\zeta$ and extinction $\eta$ of embers; and a probability of ignition $\kappa$ per landing burning ember.

Conservation equations for the mean number of fires $n$, number of embers $E$, fire perimeter $F$ and burnt area $B$ (all per unit area) follow logically from these core processes and lead to a generic mean structure and explicit formula for the spread rate $R_{\mathrm{e}}$ of a steadily spreading ember storm. These are expressed in terms of the physical parameters identified above $\left(R_{\mathrm{H}}, R_{\mathrm{F}}, R_{\mathrm{B}}, \epsilon, w, \zeta, \eta\right.$ and $\left.\kappa\right)$. In some situations these parameters might not stay constant and may vary significantly through an ember storm, in which case they should be interpreted as representing effective mean values for the modelling given here. The modelling could indeed be extended to account for such variations, as appropriate, if they were known.

As presented here, the model is not valid when spotting is relatively rare, having $\tau w$ similar in magnitude to the centroid speed $c$ or indeed the head fire spread rate $R_{\mathrm{H}}$, as indicated in Figure 3 . The interactions do not lead to an ember storm in such cases, even though they do enhance the overall fire-spread; some details of the model then need to be treated in a different manner that is not covered here.

\section{ACKNOWLEDGMENTS}

The authors particularly appreciate a suggestion of Malcolm Gill that laid the foundation for the modelling that is presented here.

\section{REFERENCES}

D.H. Anderson, E.A. Catchpole, N.J. de Mestre and T. Parkes (1982). Modeling the spread of grass fires. The ANZIAM Journal 23. 451-466.

R.A. Anthenien, S.D. Tse and A.C. Fernandez-Pello (2006). On the trajectories of embers initially elevated or lofted by small scale ground fire plumes in high winds. Fire Safety Journal 41. 349-363.

K.P. Chen and J. McAneney (2004). Quantifying bushfire penetration into urban areas in Australia. Geophysical Research Letters 31. Article No. L12212.

J.L. Consalvi, P. Mindykowski, J.P. Vantelon and B. Porterie (2011). Scaling the transport of firebrands by wind-blown plumes. Fire Safety Journal 46. 48-55.

P.F. Ellis (2000). The aerodynamic and combustion characteristics of of eucalypt bark-a firebrand study. PhD Thesis. Australian National University.

M. Gill (2005). Landscape fires as social disasters: an overview of 'the bushfire problem'. Environmental Hazards 6. 65-80 (and private communications).

R. McRae (2010) Extreme Fire - a handbook. ACT Government and Bushfire Cooperative Research Centre.

S.L. Manzello, T.G. Cleary, J.R. Shields, A. Maranghides, W. Mell, J.C. Yang (2008). Experimental investigation of firebrands: generation and ignition of fuel beds. Fire Safety Journal 43. 226-233.

B. Porterie, N. Zekri, J-P. Clerc and J-C. Loraud (2005). Influence des brandons sur la propagation d'un feu de forêt. Comptes Rendus Physique 6. 1153-1160.

G.D. Richards (1990). An elliptical growth model of forest fire fronts and its numerical solution. International Journal for Numerical Methods in Engineering 30. 1163-1179.

C.S. Tarifa, P.P. Del Notario and F.G. Moreno (1965). On the flight paths and lifetimes of burning particles of wood. Proc. Combust. Inst. 10. 1021-1037.

J.P. Woycheese, P.J. Pagni and D. Liepmann (1999). Brand propagation from large-scale fires. Journal of Fire Protection Engineering 10. 32-44. 